\title{
Formação e atitudes relacionadas às tentativas de suicídio entre profissionais de Estratégias de Saúde da Família*
}

\author{
Aline Siqueira de Almeida ${ }^{1}$ \\ (iD) https://orcid.org/0000-0002-7738-0676 \\ Kelly Graziani Glacchero Vedana ${ }^{1}$ \\ (iD) https://orcid.org/0000-0001-7363-2429
}

\begin{abstract}
* Este artigo refere-se à chamada temática "Violência autoprovocada: autolesão não suicida e comportamento suicida". Artigo extraído da tese de doutorado "Significados e atitudes relacionados ao comportamento suicida entre profissionais da Estratégia de Saúde da Família", apresentada à Escola de Enfermagem de Ribeirão Preto da Uiversidade de São Paulo, Ribeirão Preto, São Paulo, Brasil. 1 Universidade de São Paulo, Escola de Enfermagem de Ribeirão Preto, Centro Colaborador da OPAS/OMS para o Desenvolvimento da Pesquisa em Enfermagem, Ribeirão Preto, SP, Brasil.
\end{abstract}

Objetivo: investigar, entre profissionais da Estratégia Saúde da Família, associação entre a formação e as atitudes relacionadas ao comportamento suicida. Método: estudo quantitativo desenvolvido com 65 profissionais de equipes de ESF de um município de Minas Gerais. Os dados foram coletados por autoaplicação de questionário sociodemográfico e do Questionário de Atitudes Frente ao Comportamento Suicida (QUACS). Utilizada estatística descritiva, testes de associação e correlação. Resultados: a maioria dos participantes declarouse sem treinamento sobre suicídio (89\%) e negou ter lido material específico (54\%). Treinamento sobre suicídio esteve associado à maior capacidade profissional autopercebida $(p=0,017)$. Atitudes mais negativas estiveram associadas à menor percepção de capacidade profissional e a atitudes mais condenatórias. Profissionais que leram material específico sobre suicídio tiveram tanto maior capacidade profissional autopercebida $(p=0,023)$ quanto atitudes menos negativas $(p=0,042)$. Atitudes menos condenatórias foram encontradas em pessoas sem religião $(p=0,000)$, que leram sobre o suicídio $(p=0,042)$, e tiveram contato com colegas de profissão que tentaram suicídio $(p=0,013)$. Conclusão: para que o potencial da ESF na detecção de riscos, prevenção e tratamento do suicídio se traduza em ações eficazes, é importante investir em recursos de formação aos profissionais de saúde que compõem tais serviços.

Descritores: Tentativa de Suicídio; Atitude; Assistência à Saúde; Atenção Primária à Saúde.

\section{Como citar este artigo}

Almeida AS, Vedana KGG. Training and attitudes related to suicide attempts among Family Health Strategy professionals. SMAD, Rev Eletrônica Saúde Mental Álcool Drog. 2020;16(4):92-99. doi: https://dx.doi.org/10.11606/issn.1806-6976.smad.2020.165054 


\section{Training and attitudes related to suicide attempts among Family Health Strategy professionals}

Objective: to investigate, among Family Health Strategy (FHS) professionals, the association between training and attitudes related to suicidal behavior. Method: a quantitative study developed with 65 FHS team professionals from a city of Minas Gerais. Data were collected by self-application of the sociodemographic questionnaire and the Questionnaire of Attitudes to Suicidal Behavior (Questionário de Atitudes Frente ao Comportamento Suicida, QUACS). Descriptive statistics, association and correlation tests were used. Results: most of the participants reported no suicide training $(89 \%)$ and denied reading specific material (54\%). Suicide training was associated with greater self-perceived professional ability $(p=0.017)$. More negative attitudes were associated with a lower perception of professional ability and more condemnatory attitudes. Professionals who read specific material about suicide had both higher self-perceived professional skills and less negative attitudes $(p=0.023)$. Less condemnatory attitudes were found in people without a religion $(p=0.000)$, who read about suicide $(p=0.042)$, and who had contact with profession colleagues who attempted suicide $(p=0.013)$. Conclusion: in order for the potential of the FHS in risk detection, suicide prevention, and the treatment to be translated into effective actions, it is important to invest in training resources for the health professionals that make up these services.

Descriptors: Suicide Attempt; Attitude; Health Care; Primary Health Care.

\section{Capacitación y actitudes relacionadas con intentos de suicidio entre los profesionales del programa Estrategia de Salud Familiar}

Objetivo: investigar, entre los profesionales de la Estrategia de Salud Familiar, la asociación entre la capacitación y las actitudes relacionadas con el comportamiento suicida. Método: estudio cuantitativo desarrollado con 65 profesionales del equipo de ESF de una ciudad de Minas Gerais. Los datos fueron recolectados por autoaplicación del cuestionario sociodemográfico y el Cuestionario de Actitudes ante el Comportamiento Suicida (QUACS). Se utilizaron estadísticas descriptivas, pruebas de asociación y correlación. Resultados: la mayoría de los participantes informaron no haber recibido capacitación sobre suicidio (89\%)y negaron haber leído material específico (53\%). El entrenamiento suicida se asoció con una mayor capacidad profesional autopercibida $(p=0,017)$. Más actitudes negativas se asociaron con una menor percepción de la capacidad profesional y más actitudes condenatorias. Los profesionales que leyeron material específico sobre el suicidio presentaron más habilidades profesionales autopercibidas y actitudes menos negativas $(p=0,023)$. Se encontraron actitudes menos condenatorias en personas sin religión $(p=0,000)$, que leyeron sobre el suicidio $(p=0,042)$ y tuvieron contacto con colegas profesionales que intentaron suicidarse $(p=0,013)$. Conclusión: para que el potencial de la ESF en la detección de riesgos, la prevención y tratamiento del suicidio se traduzca en acciones efectivas, es importante invertir en recursos de capacitación para los profesionales de la salud que integran estos servicios.

Descriptores: Intento de Suicidio; Actitude; Cuidado de La Salud; Atención Primaria de Salud. 


\section{Introdução}

O comportamento suicida representa importante problema de saúde e pode manifestar-se por pensamentos de autodestruição, a autoagressão, tentativas de suicídio, e, finalmente, o próprio suicídio(1).

Para a Organização Mundial da Saúde (OMS), no mundo estima-se que ocorra uma morte por suicídio a cada quarenta segundos e uma tentativa a cada três segundos aproximadamente ${ }^{(1)}$. O suicídio provoca a perda de anos potenciais de vida e tem prejuízos emocionais e econômicos substanciais para os sujeitos e os serviços de saúde(2). A OMS recomenda que a prevenção do suicídio seja priorizada em programas e agendas e políticas públicas ${ }^{(1)}$.

A atenção primária à saúde possui posição privilegiada para o rastreamento e a prevenção de risco suicida. Estudo realizado na Austrália mostrou que aproximadamente $90 \%$ das pessoas que morreram por suicídio tiveram contato com um profissional de saúde nos três meses que antecederam sua morte(3).

A assistência à pessoa com comportamento suicida é essencial para salvar vidas e pode ser influenciada por uma multiplicidade de fatores entre os quais estão atitudes relacionadas ao comportamento suicida, crenças, fatores culturais, religião, formação profissional e a habilidade para avaliação de risco, planejamento e implementação dos cuidados ${ }^{(3-5)}$.

O conhecimento sobre a formação e as atitudes dos profissionais da saúde em relação ao comportamento suicida é essencial, pois sinaliza a verdadeira disposição da equipe em cuidar de pessoas em risco(6-7). No Brasil, há poucos estudos que avaliaram atitudes e a tentativa de suicídio ou o comportamento suicida de profissionais da área de saúde, identificando principalmente pesquisas envolvendo estudantes e profissionais de enfermagem ${ }^{(7-11)}$. Alguns estudos tem demonstrado que atitudes negativas relacionadas ao comportamento suicida estão associadas ao despreparo de profissionais e podem reforçar o estigma, discriminação e prejudicar o cuidado a pessoa em risco suicida(12-17).

A literatura aponta a necessidade de investigações sobre as atitudes, significados, experiências e crenças relacionadas ao comportamento suicida em diferentes $\operatorname{contextos}^{(4,18)}$. Há uma carência de estudos realizados com equipes da atenção primária, o que poderia proporcionar melhor compreensão do preparo e disposição desses profissionais para atuar na prevenção do suicídio, potencialidades, limitações e demandas na formação. Tal conhecimento pode oferecer subsídios para o planejamento de estratégias de formação acadêmica e de apoio psicossocial para os estudantes, contribuindo para a e melhoria da qualificação para a assistência. Dessa forma, este estudo investigou, entre os profissionais da Estratégia Saúde da Família, a associação entre a formação e as atitudes relacionadas ao comportamento suicida, diferenciando de outros estudos sobre a temática, por ter sido realizado com profissionais que compõem a Atenção Primária à Saúde.

\section{Método}

Estudo quantitativo realizado com 65 profissionais de 06 equipes de Estratégia Saúde da Família que compõem a rede municipal de saúde de um município do interior do estado de Minas Gerais - Brasil. Foram elegíveis para participação na pesquisa todos os profissionais (médicos, enfermeiros, técnicos de enfermagem, auxiliar de saúde bucal, dentista e agentes comunitários de saúde) que estavam inseridos no cadastro de funcionários das equipes de Estratégia de Saúde da Família do município investigado, considerando o tempo mínimo de três meses de atuação nestes serviços. Dois profissionais foram excluídos do estudo por trabalharem nos serviços investigados há menos de três meses.

A pesquisadora dirigiu-se para as unidades de saúde em dias e horários previamente agendados com o enfermeiro responsável pelo serviço. Neste momento, a equipe estaria reunida para que fossem explicados os objetivos do estudo e as orientações sobre o preenchimento dos instrumentos a serem aplicados. Foi esclarecido aos profissionais que sua participação seria voluntária, anônima e caso desistissem de participar a qualquer momento, isso seria possível sem que houvesse qualquer tipo de prejuízo para si. Todos os profissionais convidados aceitaram participar e assinaram o Termo de Consentimento Livre e Esclarecido para sua participação.

A coleta de dados ocorreu nos anos de 2016 a 2018 e se deu por meio da aplicação de questionário sociodemográfico e do Questionário de Atitudes frente ao Comportamento Suicida (QUACS) ${ }^{(10)}$. Os questionários foram autoaplicados nas unidades de saúde de cada equipe, com datas e horários previamente agendados. $A$ pesquisadora esteve presente durante o preenchimento dos questionários.

O QUACS é um instrumento brasileiro com 21 afirmações sobre atitudes. Cada afirmação é seguida por uma escala visual analógica de 10 centímetros $(\mathrm{cm})$ (10 pontos) que varia de "discordo totalmente", em uma extremidade a "concordo plenamente", em outra. O participante é convidado a indicar um ponto em cada linha que melhor represente suas atitudes. A pontuação foi computada em centímetros e os valores foram transferidos ao banco de dados com uma casa decimal. O instrumento permite a análise dos itens isoladamente e também em três fatores. O Fator 1 "sentimentos negativos diante do paciente" inclui os itens 5, 13 e 15 do questionário (maiores escores indicam mais sentimentos negativos). No Fator 2 "percepção de capacidade 
profissional" são somados os valores obtidos nos itens 1 , 10 e o item 12 com valor negativo (maiores pontuações sinalizam maior capacidade profissional autopercebida). O fator 3 "direito ao suicídio" é obtido pela soma dos itens 3, 6 e 16, sendo os dois últimos itens considerados com valores invertidos (e maiores pontuações indicam uma atitude menos condenatória) ${ }^{(10)}$.

Foram construídos dois Questionários Sociodemográficos, sendo um deles direcionado aos profissionais com ensino superior ou técnico e outro para os agentes comunitários de saúde (ACS). Os questionários continham informações sobre idade, sexo (feminino/masculino), atuação profissional (médico, enfermeiro, dentista, técnico/auxiliar em enfermagem, auxiliar de saúde bucal), formação (Técnico/Profissionalizante, Superior incompleto, Superior completo, Especialização/Residência, Mestrado, Doutorado), treinamento ou experiência profissional diretamente relacionada à saúde mental (sim ou não), tempo de exercício na profissão (anos completos), tempo de exercício na unidade (anos completos), contato com pessoa que tentou suicídio ( $\operatorname{sim}$ ou não) e se já leu material específico sobre suicídio (sim ou não).

Utilizou-se estatística descritiva para a apresentação das variáveis sociodemo-gráficas, educacionais e relacionadas às atitudes frente ao comportamento suicida. Posteriormente, aplicados os testes de normalidade Kolmogorov-Smirnova e Shapiro-Wilk para direcionar a opção por testes paramétricos ou não paramétricos. Foram utilizados testes de comparação de médias (teste T e teste de Mann- Whitney) para avaliar associações entre as variáveis categóricas e os valores obtidos nos fatores do QUACS.O teste de correlação de Pearson foi utilizado para avaliar as correlações existentes entre as variáveis quantitativas e os escores dos fatores do QUACS. A correlação de Spearman foi utilizada para avaliar as variáveis não paramétricas (idade e Q 21). O valor de alfa mínimo considerado foi de $p<0,05$ em todos os testes.

O estudo seguiu as recomendações sobre pesquisas com seres humanos e foi submetido e aprovado pelo Comitê de Ética e Pesquisas da Escola de Enfermagem de Ribeirão Preto, com número da aprovação e regulamentação CAAE:56529816.0.0000.5393.

\section{Resultados}

Neste estudo foram analisados dados obtidos por meio de dois questionários aplicados aos participantes para levantamento de dados sociodemográficos, um para os profissionais de saúde e outro para os agentes comunitários de saúde.

O estudo foi desenvolvido com 65 profissionais da ESF, sendo que 29 (45\%) são profissionais da saúde: enfermeiros $(8 \%)$, médicos $(20 \%)$, técnicos e auxiliares de enfermagem ( $11 \%)$, outras categorias (6\%). Considerando o tempo de experiência profissional, $23 \%$ deles possuem tempo de experiência profissional menor ou igual 8 meses; $30 \%$ possuem entre 1-5 anos de experiência; $27 \%$ possuem entre 6-15 anos de experiência e $20 \%$ possuem entre 16-30 anos de experiência. Participaram do estudo 36 (55\%) Agentes Comunitários de Saúde, com tempo de experiência variado. A maioria (61\%) entre $2-10$ anos, $11 \%$ entre 11-13 anos e $28 \%$ com menos de 01 ano de experiência. Predominaram pessoas do sexo feminino (86\%), com idade entre 20-59 anos (95\%), com religião (92\%), com contato com alguém que tentou suicídio (78\%). A maioria dos profissionais negou treinamento prévio sobre prevenção do suicídio ( $89 \%$ ) ou leituras sobre o tema (54\%), negou casos de suicídio na família ( $81 \%)$ ou contato com colegas de profissão que tentaram suicídio ( $81 \%)$.

A Tabela 1 apresenta as associações entre características sociodemográficas, experiência e treinamento profissional dos participantes e as atitudes relacionadas ao comportamento suicida, avaliadas por meio do Questionário de Atitudes Frente ao Comportamento Suicida (QUACS). No fator 1, que indica atitudes mais negativas em relação ao suicídio, mulheres tiveram maiores pontuações $(p=0,044)$, e também os profissionais que não tiveram leitura de material específico sobre suicídio $(p=0,001)$, que possuem religião $(p=0,010)$ e que não tiveram contato com colegas de profissão que tentaram suicídio $(p=0,001)$ (Tabela 1).

O fator 2 indica maior percepção de capacidade profissional, e obtiveram maiores pontuações profissionais que tiveram treinamento sobre suicídio $(p=0,017)$, contato com pessoa que tentou suicídio $(p=0,013)$, leitura de material específico sobre suicídio $(p=0,023)$ e contato com colegas de profissão que tentaram suicídio $(p=0,013)$ (Tabela 1$)$.

O fator 3 se refere a atitudes menos condenatórias em relação ao suicídio, tendo maiores pontuações os profissionais que tiveram leitura de material específico sobre suicídio $(p=0,042)$, que não tinham religião $(p=0,000)$ e que tiveram contato com colegas de profissão que tentaram suicídio $(p=0,001)$ (Tabela 1 ).

Em relação à Questão 21 do questionário (QUACS), que avalia os pensamentos suicidas, os resultados de testes não demonstraram associação estatisticamente significante entre a Questão 21 e as variáveis categóricas do estudo.

De acordo com os dados apresentados na Tabela 2, observa-se uma correlação negativa fraca ( $r=-$ 0,159 ) entre o Fator 1 e o Fator 2 do QUACS, o que significa que quanto maior a percepção de capacidade profissional, menores os sentimentos negativos em 
relação aos pacientes com comportamento suicida. O mesmo se observa entre os Fator 1 e o Fator 3 do QUACS $(r=-0,447)$ e $(p=0,00)$ quanto mais sentimentos negativos, menor percepção do direito ao suicídio. Entre o Fator 1 e a Questão 21 do QUACS também houve uma correlação negativa fraca $(r=-0,045)$, indicando que os profissionais que já pensaram em se matar tiveram menos sentimentos negativos em relação a pessoas com comportamento suicida. Entre idade e o Fator 3 também foi observada uma correlação negativa fraca $(r=-0,197)$, representando que quanto maior a idade menor a percepção do direito ao suicídio.

Tabela 1 - Características sociodemográficas, experiência e treinamento profissional dos participantes do estudo segundo os fatores do Questionário de Atitudes Frente ao Comportamento Suicida (QUACS $(n=65)$. Borda da Mata, MG, Brasil, 2016-2018

\begin{tabular}{|c|c|c|c|c|c|c|c|c|c|}
\hline \multirow{2}{*}{ Variáveis } & \multicolumn{3}{|c|}{ Fator 1-Sentimentos negativos } & \multicolumn{3}{|c|}{ Fator 2-Capacidade profissional } & \multicolumn{3}{|c|}{ Fator 3-Direito ao Suicídio } \\
\hline & $\mathbf{M}^{*}$ & $\mathbf{D}^{\dagger}$ & $\mathbf{p}^{\ddagger}$ & $\mathbf{M}^{*}$ & $\mathbf{D}^{\dagger}$ & $\mathbf{p}^{\ddagger}$ & $\mathbf{M}^{*}$ & $\mathbf{D}^{\dagger}$ & $\mathbf{p}^{\ddagger}$ \\
\hline \multicolumn{10}{|l|}{ Sexo } \\
\hline Feminino & 12,9 & 6,3 & $0,04^{\S}$ & 13,6 & 5,4 & $0,763 \|$ & 7,8 & 6,3 & $0,47 \|$ \\
\hline Masculino & 8,3 & 3,9 & & 14,2 & 8,3 & & 14,4 & 9,4 & \\
\hline \multicolumn{10}{|l|}{ Treinamento sobre suicídio } \\
\hline Sim & 12,1 & 8,9 & $0,958^{\S}$ & 18,5 & 5,7 & $0,017 \|$ & 12,3 & 8,9 & $0,189 \|$ \\
\hline Não & 12,2 & 5,9 & & 13,0 & 5,5 & & 8,2 & 6,8 & \\
\hline \multicolumn{10}{|c|}{ Contato com pessoa que tentou suicídio } \\
\hline Sim & 12,0 & 6,6 & $0,631^{\S}$ & 14,5 & 5,4 & $0,013 \|$ & 9,4 & 7,3 & $0,110^{\|}$ \\
\hline Não & 12,9 & 4,5 & & 10,3 & 5,7 & & 6,1 & 5,9 & \\
\hline \multicolumn{10}{|c|}{ Leitura de material específico } \\
\hline Sim & 9,6 & 5,0 & $0,001^{\S}$ & 15,4 & 6,0 & $0,023 \|$ & 10,8 & 7,8 & 0,042 \\
\hline Não & 14,5 & 6,2 & & 12,1 & 5,1 & & 6,8 & 5,9 & \\
\hline \multicolumn{10}{|l|}{ Religião } \\
\hline Sim & 12,8 & 6,0 & $0,01^{\S}$ & 13,3 & 5,6 & $0,126 \|$ & 7,6 & 6,2 & 0,000 \\
\hline Não & 5,4 & 3,9 & & 17,4 & 6,8 & & 20,8 & 5,3 & \\
\hline \multicolumn{10}{|l|}{ Caso de suicídio na família } \\
\hline Sim & 12,8 & 4,5 & $0,714 \S$ & 12,4 & 9,0 & $0,419 \| 1$ & 8,8 & 8,5 & $0,780^{\|}$ \\
\hline Não & 12,1 & 6,5 & & 13,9 & 4,8 & & 8,6 & 6,8 & \\
\hline \multicolumn{10}{|c|}{$\begin{array}{l}\text { Colegas de profissão que tentaram } \\
\text { suicídio }\end{array}$} \\
\hline $\operatorname{Sim}$ & 6,9 & 3,9 & $0,001^{\S}$ & 17,3 & 5,8 & $0,013^{\|}$ & 15,3 & 7,3 & $0,001^{\|}$ \\
\hline Não & 13,4 & 6,0 & & 12,8 & 5,4 & & 7,1 & 6,1 & \\
\hline
\end{tabular}

$\mathrm{M}^{*}=$ Média; $\mathrm{D}^{+}=$Desvio Padrão; $\mathrm{p}^{+}=$Valor de $\mathrm{p}$; ${ }^{\S}$ Teste- $\mathrm{t}$; "Teste $\mathrm{U}$ de Mann-Whitney

Tabela 2 - Teste de correlação entre os fatores do QUACS e o escore obtido na questão 21 do QUACS e a idade dos participantes ( $n=63$ ). 2016-2018. Borda da Mata, MG, Brasil, 2016-2018

\begin{tabular}{lccc}
\hline \multicolumn{1}{c}{ Variáveis } & Fator 1-Sentimentos negativos & Fator 2-Capacidade profissional & Fator 3-Direito ao Suicídio \\
\cline { 2 - 4 } & $\mathbf{p}^{*}(\mathbf{r})^{\dagger}$ & $\mathbf{p}^{*}(\mathbf{r})^{\dagger}$ & \\
\hline Fator 1 - Sentimentos negativos & $(1)$ & 1 & $\mathbf{p}^{*}(\mathbf{r})^{\dagger}$ \\
Fator 2 - Capacidade profissional & $0,204^{*}(-0,159)^{\S}$ & $0,15^{*}(0,299)^{\S}$ & 1 \\
Fator 3 - Direito ao Suicídio & $0,000^{*}(-0,447)^{\S}$ & $0,073^{*}(0,224)^{\S}$ & $0,005^{*}(0,346)^{\|}$ \\
Q21- Pensamentos suicidas & $0,721^{*}(-0,045)^{\S}$ & $0,373^{*}(0,114)^{\S}$ & $0,121^{*}(-0,197)^{\|}$ \\
Idade & $0,012^{*}(0,316)^{\S}$ &
\end{tabular}






\section{Discussão}

No presente estudo, foram investigadas associações entre atitudes relacionadas ao comportamento suicida e características sociodemográficas. A maioria dos entrevistados era mulher e essa característica esteve associada a atitudes mais negativas. Na literatura, encontra-se uma variedade de resultados relacionados às atitudes frente ao comportamento suicida e fatores sociodemográficos, considerando estudos com estudantes e profissionais em sua prática. Estudos que identificaram mulheres com atitudes menos moralistas ${ }^{(19)}$ outros estudos não identificam diferença em atitudes relacionadas ao sexo(14,20) e também estudos que encontraram atitudes mais moralistas em mulheres ${ }^{(8-9)}$. Esta questão é heterogênea na literatura e carece de maior investigação.

Pessoas mais velhas tiveram atitudes mais condenatórias em relação ao comportamento suicida. A literatura apresenta resultados heterogêneos sobre a relação entre a idade e a atitude frente ao comportamento suicida ${ }^{(12,14,17)}$. Neste estudo, a maioria dos profissionais afirmou ter religião e possuir religião foi uma característica associada a atitudes mais negativas e mais condenatórias. A literatura apresenta resultados heterogêneos com relação à religião e atitudes frente ao comportamento suicida( $(7,10)$.

Neste estudo, a maioria dos participantes referiu não ter treinamento sobre prevenção do suicídio e referiu nunca ter lido material específico sobre o assunto, embora tivesse tido contato com alguém que tentou suicídio. A exposição educacional relacionada ao tema não parece ser uma especificidade da atenção primária, pois é corroborada por estudo brasileiro realizado com estudantes de enfermagem ${ }^{(9)}$ e também foi identificada entre profissionais de serviços de emergência brasileiros(8). Por outro lado, um estudo espanhol identificou que a maioria dos enfermeiros tinha treinamento sobre prevenção do comportamento suicida(19). A literatura aponta a importância de investigar os fatores ligados à baixa exposição educacional sobre o comportamento suicida no Brasil(8), considerando a relevância do assunto no cenário mundial e as diferenças na formação de profissionais sobre o tema em diferentes contextos.

Diversos estudos têm demonstrado que a falta de preparo e treinamento dos profissionais está associada a atitudes negativas ${ }^{(5,12,19-20)}$ enquanto a formação profissional está associada a atitudes positivas em relação ao comportamento suicida(8-9) e proporciona clareza e confiança em relação ao papel do profissional no atendimento(20).

No presente estudo, o treinamento sobre suicídio esteve associado à maior percepção da própria capacidade para o cuidado. Esse resultado corrobora com estudo brasileiro que encontrou que diferentes formas de treinamento (disciplina, aula ou laboratório) estiveram associadas à percepção da competência

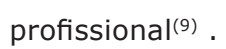

A leitura de material específico sobre suicídio esteve associada tanto à maior percepção da capacidade profissional quanto a atitudes menos negativas. Foi identificado ainda que, as atitudes mais negativas frente ao comportamento suicida estiveram associadas à menor percepção de capacidade profissional e a atitudes mais condenatórias, demonstrando que as atitudes mensuradas pelo instrumento QUACS estiveram integradas entre si.

A promoção de atitudes positivas, empáticas e seguras entre os profissionais é importante pois, apesar de existirem evidências de atitudes positivas dos profissionais de saúde em relação à pessoa com comportamento suicida(21), a maioria dos estudos evidencia uma predominância de atitudes negativas que afetam os cuidados prestados a pessoas em risco de suicídio(12,21).

Neste estudo, a maioria dos profissionais referiu ter tido contato com alguém que tentou suicídio, mas negou casos de suicídio na família ou comportamento suicida entre os colegas de profissão. O contato com pessoas com comportamento suicida foi associado à maior percepção da capacidade profissional, conforme já identificado em uma investigação brasileira ${ }^{(8)}$.

Destaca-se ainda que, no presente estudo, o contato com colegas de profissão que tentaram suicídio foi associado a atitudes menos condenatórias e além de ser associado à maior percepção da capacidade profissional. É possível que o contato com alguém com comportamento suicida possa oportunizar aprendizado, reflexão e redefinição da própria capacidade para cuidar. A atitude compreensiva e empática é um fator importante na prevenção ao suicídio ${ }^{(19,22-23)}$ que precisa ser abordado na formação de profissionais de saúde(24).

\section{Conclusão}

O presente estudo investigou, entre profissionais da ESF, a associação entre a formação e as atitudes relacionadas ao comportamento suicida e identificou que, apesar da baixa exposição educacional ao suicídio, a maioria dos participantes teve contato com alguém que tentou suicídio. O treinamento sobre suicídio esteve associado à maior capacidade profissional autopercebida.

As atitudes mais negativas frente ao comportamento suicida estiveram associadas à menor percepção de capacidade profissional e a atitudes mais condenatórias. Os profissionais que leram material específico sobre suicídio tiveram tanto maior capacidade profissional autopercebida quanto atitudes menos negativas. 
No que se refere às associações entre atitudes e experiências e características sociodemográficas, as atitudes menos condenatórias foram encontradas em pessoas sem religião que tiveram contato com colegas de profissão que tentaram suicídio. Pessoas mais velhas tiveram atitudes mais condenatórias em relação ao comportamento suicida. Mulheres e pessoas com menos pensamentos suicidas ao longo da vida tiveram atitudes mais negativas.

Este estudo permitiu aprofundamento no conhecimento sobre atitudes dos profissionais da atenção primária sobre o comportamento suicida e encontrou que as atitudes estão associadas a características pessoais e formação profissional. Desse modo, é importante desenvolver e avaliar ações de formação continuada que possam promover melhores atitudes.

As principais limitações deste estudo foram o uso de uma amostra de conveniência reduzida pertencente a um único município e o delineamento transversal. É recomendável que futuras análises investiguem as questões abordadas neste estudo em diferentes contextos. Contudo, esta pesquisa contribui de forma significativa para a melhor compreensão das atitudes e significados relacionados ao comportamento suicida entre profissionais que atuam na Atenção Primária à Saúde.

\section{Referências}

1.World Health Organization (WHO). Preventing suicide: a global imperative. 2014. [cited Jun 21, 2020]. Available from: http://apps.who.int/iris/ bitstream/10665/131056/8/9789241564878_eng. pdf?ua $=1 \& u a=1$

2. World Health Organization (WHO). Comprehensive mental health action plan 2013-2020. Geneva; 2013. [cited Jun 21, 2020]. Available from: https://apps.who.int/ iris/bitstream/handle/10665/89966/9789241506021_ eng.pdf;jsessionid=b6677c6c0787bf9cba94d71f2f6e38 54 ? sequence $=1$

3.De Leo D, Draper BM, Snowdon, J,Kolves, K. Contacts with health professionals before suicide: missed opportunities for prevention? Comprehensive Psychiatry. 2013;54(7);1117-23. doi: 10.1016/j. comppsych.2013.05.

4.Nebhinani M, Nebhimani N, Tamphasana L, Gaikwad AD. Nursing students' attitude towards suicide attempters: a study from rural part of northern India. J Neurosci Rural Practice. 2013;10(4);400-7. doi: 10.4103/0976-3147.120240

5.Srivastava M, Tiwari R. A comparative study of attitude of mental health versus non mental professionals toward suicide indian. Indian J Psychol Medicine. 2012.11(34):66-9. doi: 10.4103/0253-7176.96163 6.Magalhães CA, Neves DMM, Brito LMDM, Leite BBC, Pimenta MMF, Vidal CEL. Atitudes de estudantes de medicina em relação ao suicídio. Rev Bras Educ Médica. 2014;38(4):470-6. doi 10.1590/ S0104-42301998000200012

7.Storino BD, Campos CF, Chicata LCO, Campos MA, Matos MSC, Nunes RMCM, et al. Atitudes de profissionais da saúde em relação ao comportamento suicida. Cad Saúde Coletiva. 2018;26(4):369-77. doi: 10.1590/1414-462X201800040191

8. Vedana KGG, Magrini DF, Zanetti ACG, Miasso AI, Borges TL, Santos AM. Attitudes towards suicidal behaviour and associated factors among nursing professionals: a quantitative study. J Psych Mental Health Nurs. 2017;24(9):651-9.doi: 10.1111/jpm.12413.

9. Moraes SM, Magrini DF, Zanetti ACG, Santos AM, Vedana KGG. Attitudes and associated factors related to suicide among nursing undergraduates. Acta Paul Enferm. 2016.29(6):643-9. doi. org/10.1590/1982-0194201600090

10.Botega NJ, Barros MBA, Oliveira HB, Dalgalarrondo $\mathrm{P}$, Marín-León L. Suicidal behavior in the community: prevalence and factors associated with suicidal ideation. Rev Bras Psiquiatr. 2005;27(1):45-53. doi.org/10.1590/ S1516-44462005000100011

11.Botti NCL, Araújo LMC, Costa EE, Machado JSA. Nursing students attitudes across the suicidal behavior. Invest Educ Enferm. 2015;33(2):334-42. doi. org/10.1590/S1516-44462005000100011

12. Karman $P$, Kool N, Poslawsky IE, van Meijel B. Nurses' attitudes towards self-harm: A literature review. J Psychiatr Ment Health Nurs. 2015;22(1):65-75. doi.10.1111/jpm. 12171

13. Saunders KEA, Hawton K, Fortune S, Farrell S. Attitudes and knowledge of clinical staff regarding people who self-harm: A systematic review. J Affect Disorders. 2012. doi: 10.1016/j.jad.2011.08.024.

14. McCarthy L, Gijbels H. An examination of emergency department nurses' attitudes towards deliberate selfharm in an Irish teaching hospital. Int Emerg Nurs. 2010; doi: 10.1016/j.ienj.2009.05.005.

15. Kelly M, McCarthy S, Sahm LJ. Knowledge, attitudes and beliefs of patients and carers regarding medication adherence: a review of qualitative literature. Eur J Clin Pharmacol [Internet]. 2014;70(12):1423-31. Available from: http://www.ncbi.nlm.nih.gov/pubmed/25277162 16.Santos JCS, Pereira RM, Erse MPQ de A, Façanha JDN, Marques LAFA, Rosa MP, et al. Impact of "+Contigo" training on the knowledge and attitudes of health care professionals about suicide. Rev. Latino-Am. Enferm. 2014;22(4):679-84. doi. org/10.1590/0104-1169.3503.2467.

17.Conlon M, O' Tuathail C. Measuring emergency department nurses' attitudes towards deliberate self-harm using the Self-Harm Antipathy Scale. 
Int Emerg Nurs. 2012;20(1):3-13. doi: 10.1016/j. ienj.2010.08.001.

18.Talseth AG, Gilje F. Unburdening suffering: responses of psychiatrists to patients' suicide deaths. Nurs Ethics. 2007;14(5):620-36. doi: 10.1177/0969733007080207 19.Carmona-Navarro, MC, Pichardo-Martínez, MC. Attitudes of nursing professionals towards suicidal behavior: Influence of emotional intelligence Rev. Latino-Am. Enferm. 2012;20(6):1161-8. doi: 10.1590/ s0104-11692012000600019

20.Ramberg IL, Di Lucca, MA, Hadlaczky, G. The Impact of Knowledge of Suicide Prevention and Work Experience among Clinical Staff on Attitudes towards Working with Suicidal Patients and Suicide Prevention. Int J Environ Res Public Health. 2016;13(2):195. doi: 10.3390/ ijerph13020195

21.Ouzouni C, Nakakis K. Nurses' attitudes towards attempted suicide. Health Sci J. [Internert]. 2013 [cited Ago 26, 2020];7(1):119-34. Available from: http://www.hsj.gr/medicine/nurses-attitudes-towardsattempted-suicide. php?aid $=3054$

22. Organização mundial da Saúde (OMS). Prevenção do suicídio: Um manual para profissionais da saúde em atenção primária. Transtornos mentais e comportamentais. Genebra: Departamento de Saúde Mental; 2000. [Acesso 21 jun 2020]. Disponível em: http://www.who.int/mental_health/prevention/suicide/ en/suicideprev_phc_port.pdf

23. Ministério da Saúde. Secretaria de Atenção a Saúde. Departamento de Atenção Básica (BR). Saúde mental. Cadernos de Atenção Básica, n.34:173. Brasília: Ministério da Saúde; 2013. [Acesso 21 jun 2020]. Disponível em: http://189.28.128.100/dab/docs/ portaldab/publicacoes/caderno_34.pdf

24.Santos JC. Suicide: can we prevent the most mysterious act of the human being? Rev Port Enferm Saúde Mental. [Internet]. 2015 [cited Jun 21 2020];2:7-8. Disponível em: http://www. scielo.mec.pt/scielo.php?script $=$ sci_arttext\&pi $\mathrm{d}=$ S1647-21602015000100001.

\section{Contribuição dos autores}

Concepção e planejamento do estudo: Aline Siqueira de Almeida e Kelly Graziani Giacchero Vedana. Obtenção dos dados: Aline Siqueira de Almeida. Análise e interpretação dos dados: Aline Siqueira de Almeida e Kelly Graziani Giacchero Vedana. Análise estatística: Aline Siqueira de Almeida e Kelly Graziani Giacchero Vedana. Redação do manuscrito: Aline Siqueira de Almeida e Kelly Graziani Giacchero Vedana. Revisão crítica do manuscrito: Aline Siqueira de Almeida.

Todos os autores aprovaram a versão final do texto. Conflito de interesse: os autores declararam que não há conflito de interesse. 